\title{
Hypoxia enhances the wound-healing potential of adipose-derived stem cells in a novel human primary keratinocyte-based scratch assay
}

\author{
SIMONE RIIS $^{1}$, RHONDA NEWMAN ${ }^{2}$, HILAL IPEK ${ }^{1,3}$, JENS I. ANDERSEN ${ }^{1}$, DAVID KUNINGER $^{2}$, \\ SHAYNE BOUCHER ${ }^{2}$, MOHAN C. VEMURI ${ }^{2}$, CRISTIAN P. PENNISI ${ }^{1}$, VLADIMIR ZACHAR $^{1}$ and TRINE FINK ${ }^{1}$ \\ ${ }^{1}$ Laboratory for Stem Cell Research, Department of Health Science and Technology, Aalborg University, Aalborg, \\ Denmark; ${ }^{2}$ Thermo Fisher Scientific, Frederick, MD, USA; ${ }^{3}$ Faculty of Medicine, Ege University, Izmir, Turkey
}

Received August 31, 2016; Accepted January 25, 2017

DOI: $10.3892 /$ ijmm.2017.2886

\begin{abstract}
Preclinical studies have suggested that paracrine factors from adipose-derived stem cells (ASCs) promote the healing of chronic wounds, and that the exposure of ASCs to hypoxia enhances their wound healing effect. To aid the translation of these findings into clinical use, robust wound models are necessary to explore each aspect of wound healing. The aspect of re-epithelization is often studied in a scratch assay based on transformed keratinocytes. However, there are concerns regarding the validity of this model, since these cell lines differ from normal keratinocytes, both in terms of proliferative capacity and differentiation, and sensitivity to environmental cues. In this study, the main challenge of using primary keratinocytes to examine the effects of ASCs was identified to be their different requirements for calcium in the culture media. We confirmed that a high calcium content led to morphological and cytoskeletal changes in primary keratinocytes, and demonstrated that a low calcium content compromised the growth of ASCs. We found that it is possible to perform the wound healing assay with primary keratinocytes, if the conditioned media from the ASCs is dialyzed to reduce the calcium concentration. Additionally, using this model of re-epithelization, conditioned media from normoxic ASCs was shown to markedly increase the rate of wound closure by primary keratinocytes, and this effect was
\end{abstract}

Correspondence to: Dr Trine Fink, Laboratory for Stem Cell Research, Department of Health Science and Technology, Aalborg University, Fredrik Bajers Vej 3B, DK-9220 Aalborg Ø, Denmark

E-mail: trinef@hst.aau.dk

Abbreviations: ASCs, adipose-derived stem cells; bFGF, basic fibroblast growth factor; EGF, epidermal growth factor; HGF, hepatocyte growth factor; IGF-1, insulin-like growth factor-1; KGF, keratinocyte growth factor; TCP, tissue culture polystyrene

Key words: adipose-derived stem cells, primary keratinocytes, wound healing, scratch assay, calcium, hypoxia significantly enhanced with media from the hypoxia-exposed ASCs. These findings, which are in line with the observations from previous in vivo studies, highlight the validity of this modified assay to investigate the wound healing properties of ASCs in vitro.

\section{Introduction}

It has been proposed that adipose-derived stem cells (ASCs) can be used for the treatment of chronic wounds (1). Several preclinical studies have demonstrated that ASCs secrete soluble factors that promote angiogenesis and keratinocyte re-epithelization, and protect against apoptosis (2-4). Furthermore, studies have demonstrated that the culture of ASCs under hypoxic conditions enhances their angiogenic properties $(2,5)$; however, the mechanisms through which hypoxia modulates the ASC properties relevant to re-epithelialization have not yet been fully elucidated.

A commonly used in vitro model to assess wound healing is the scratch assay, which is based on creating a small scratch in a confluent monolayer cell culture and monitoring the closure of the scratch by migration/growth of the cells (Fig. 1A). To mimic the wound healing process of cutaneous wounds, keratinocytes are the most relevant cells, as re-epithelization steps include sequential keratinocyte proliferation, migration and differentiation converging in stratification (6).

Previously, when assessing the wound healing effect of ASCs and other mesenchymal stem cells on keratinocytes in a scratch assay, a combination of conditioned media from stem cells and the spontaneously transformed aneuploid immortal keratinocyte cell line, HaCaT, has often been preferred $(3,7,8)$. However, as transformed cells often display an altered response to growth factors and cytokines compared to their non-cancerous counterparts (9), and may respond in a hyperactive manner to hypoxia-induced factors, such as epidermal growth factor (EGF) and basic fibroblast growth factor (bFGF) (9-11), this cell line may not be the best option to predict in vivo responses. Another difference between primary keratinocytes and the $\mathrm{HaCaT}$ cell line is that primary keratinocytes are sensitive to calcium concentrations $>90 \mu \mathrm{M}(12)$, which induces differentiation and the cessation of proliferation, 
whereas $\mathrm{HaCaT}$ cells are relatively insensitive to variations in calcium concentration (13). The calcium-sensitivity of primary keratinocytes may provide an explanation for the favored use of $\mathrm{HaCaT}$ cells when evaluating the effects of conditioned media from ASCs on wound healing, as ASCs routinely are grown in media with a calcium concentration of $1 \mathrm{mM}$, far surpassing what is tolerated by keratinocytes (14).

In this study, we describe how the ASC-keratinocyte scratch assay can be modified to better mimic in vivo re-epithelialization. This modification entails dialysis of conditioned media from ASCs prior to testing on primary keratinocytes. Furthermore, we demonstrate that the modified assay can be used to explore the effects of hypoxia on the wound healing properties of ASCs.

\section{Materials and methods}

Cell culture. Human primary keratinocytes from three donors; HEKa lot \#1249380, HEKa lot \#1352914, HEKn lot \#1030422 (Thermo Fisher Scientific, Frederick, MD, USA) were used for all the experiments. They were maintained in EpiLife, composed of EpiLife ${ }^{\circledR}$ basal medium $\left(\right.$ Gibco $^{\mathrm{TM}} /$ Thermo Fisher Scientific, Taastrup, Denmark) supplemented with 1X Human Keratinocyte Growth Supplement (Gibco ${ }^{\mathrm{TM}}$ / Thermo Fisher Scientific), $100 \mathrm{U} / \mathrm{ml}$ penicillin, $0.1 \mathrm{mg} /$

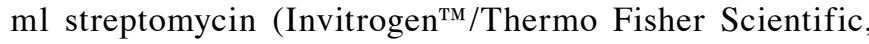
Taastrup, Denmark). The keratinocytes were maintained as prescribed by the manufacturer, and cultured on tissue culture polystyrene (TCP) flasks (Greiner Bio-One, Fredensborg, Denmark) coated with Coating Matrix kit. For subcultivation, TrypLE ${ }^{\mathrm{TM}}$ (both from Gibco/Thermo Fisher Scientific) was used.

The ASCs used in this study (ASC21) have previously been isolated and extensively characterized in our laboratory (2,15-19). The cells were obtained from the adipose tissue of a healthy donor using a protocol that was approved by the Regional Committee on Biomedical Research Ethics of Northern Jutland, Denmark (project no. VN 2005/54). The ASCs were cultured in StemPro, composed of StemPro ${ }^{\circledR}$ MSC SFM XenoFree (Gibco/Thermo Fisher Scientific) supplemented with $200 \mathrm{mM}$ L-glutamine and $100 \mathrm{U} / \mathrm{ml}$ penicillin, $0.1 \mathrm{mg} / \mathrm{ml}$ streptomycin (both from Gibco/Thermo Fisher Scientific) and cultured on cultured on TCP flasks (Greiner Bio-One), which were coated with CellStart ${ }^{\mathrm{TM}}$ CTS $^{\mathrm{TM}}$ according to the manufacturer instructions. For subcultivation, TrypLE ${ }^{\mathrm{TM}}$ (both from Gibco/Thermo Fisher Scientific) was used.

Comparison of ASC and keratinocyte morphology and growth with varying concentrations of calcium. To compare the effects of the different calcium concentrations in EpiLife and StemPro on keratinocytes and ASCs, the cells were seeded at a density of 50,000 and 8,000 cells $/ \mathrm{cm}^{2}$, respectively in 96-well plates and cultured until $80 \%$ confluent. At this point, the cells were washed with phosphate-buffered saline (PBS; Gibco ${ }^{\mathrm{TM}} /$ Thermo Fisher Scientific) and supplied with either EpiLife, EpiLife with increasing levels of $\mathrm{CaCl}_{2}$ from $60 \mu \mathrm{M}$ to $2 \mathrm{mM}$, or StemPro, and incubated for $24 \mathrm{~h}$. To assess cell morphology and cell number, the cells were washed with PBS and fixed in $4 \%$ formaldehyde, stained with Hoechst $33342\left(1 \mu \mathrm{g} / \mathrm{ml}\right.$; Molecular Probes ${ }^{\circledR}$, Eugene, OR, USA), permeabilized using $0.1 \%$ Triton X-100
(Sigma-Aldrich), and stained with BODIPY ${ }^{\circledR}$ 558/568 phalloidin (1:40; Molecular Probes). The stained cells were kept in PBS at $4^{\circ} \mathrm{C}$ until analysis. Fluorescence images were obtained using the AxioVision software package with a Zeiss AxioObserver.Z1 microscope equipped with an AxioCam MRm camera and a motorized stage (Carl Zeiss, Oberkochen, Germany). To quantify the cell number, the number of nuclei was counted using ImageJ $1.47 \mathrm{v}$ (National Institutes of Health, Bethesda, MD, USA).

To evaluate the effect on keratinocytes of lowering the calcium concentration of StemPro, the medium was dialyzed against EpiLife basal medium. For dialysis, $10 \mathrm{ml}$ StemPro were injected into a Slide-A-Lyzer Dialysis Cassette, 2 MWCO (Pierce $^{\mathrm{TM}} /$ Thermo Fisher Scientific) and dialyzed in 1.25 liters EpiLife at $4^{\circ} \mathrm{C}$ for $2 \mathrm{~h}$ after which the EpiLife was exchanged with new EpiLife (1.25 liters) for continuing dialysis overnight. After dialysis, the low-calcium StemPro was tested on keratinocyte morphology as described above.

Effect of combining EpiLife and StemPro on keratinocyte morphology. To determine whether diluting StemPro into EpiLife improves the compatibility with keratinocytes, different ratios of EpiLife vs. StemPro were tested on the keratinocytes. The keratinocytes were seeded at 20,000 cells/ $\mathrm{cm}^{2}$ in 96-well plates and incubated overnight, after which, the cells were washed with PBS and cultured for $24 \mathrm{~h}$ in either EpiLife, StemPro, or EpiLife and StemPro at the ratios 3:1, 1:1 and 1:3. The morphology of the cells was evaluated by phase contrast using the IncuCyte $\mathrm{ZOOM}^{\circledR}$ system (Essen BioScience, Hertfordshire, UK) and IncuCyte ${ }^{\mathrm{TM}}$ High Definition Imaging Mode.

To evaluate whether EpiLife supplemented with the protein fraction from conditioned StemPro supports normal keratinocyte morphology, conditioned StemPro was concentrated in 3,000 NMWL Amicon Ultra-15 centrifugal filter units (Merck Millipore, Darmstadt, Germany) and reconstituted in EpiLife to 50,75 or $100 \%$ of the original concentration and tested on the keratinocytes as described above.

Preparation of conditioned media. For the production of conditioned media, the ASCs were seeded in T175 culture flasks at a density of 8,000 cells $/ \mathrm{cm}^{2}$, and incubated in a standard incubator at $37^{\circ} \mathrm{C}, 20 \% \mathrm{O}_{2}, 5 \% \mathrm{CO}_{2}$. When the cells were $80 \%$ confluent, they were washed twice in PBS and $30 \mathrm{ml}$ fresh StemPro were added to each flask. Half of the flasks were left in the standard incubator for normoxic conditioning of the media and the other half were transferred to a BioSpherix clove box (Xvivo; BioSpherix, Redfield, NY, USA) and cultured at $37^{\circ} \mathrm{C}, 1 \% \mathrm{O}_{2}, 5 \% \mathrm{CO}_{2}$ for hypoxic conditioning. After $24 \mathrm{~h}$ of incubation the media were harvested, centrifuged to pellet debris, and the supernatant kept at $-80^{\circ} \mathrm{C}$ until further analysis.

Scratch assay. To investigate the wound healing effect of ASCs on human primary keratinocytes, the keratinocytes were seeded with 50,000 cells $/ \mathrm{cm}^{2}$ in $24-$ or 96 -well plates. When the cells formed a confluent monolayer, they were scratched using the Wounding Pin Tool (V\&P Scientific, Inc., San Diego, CA, USA) or the WoundMaker ${ }^{\mathrm{TM}}$ (Essen BioScience) and washed in PBS to remove cell debris. StemPro, dialyzed StemPro, conditioned StemPro, dialyzed normoxic/hypoxic conditioned 


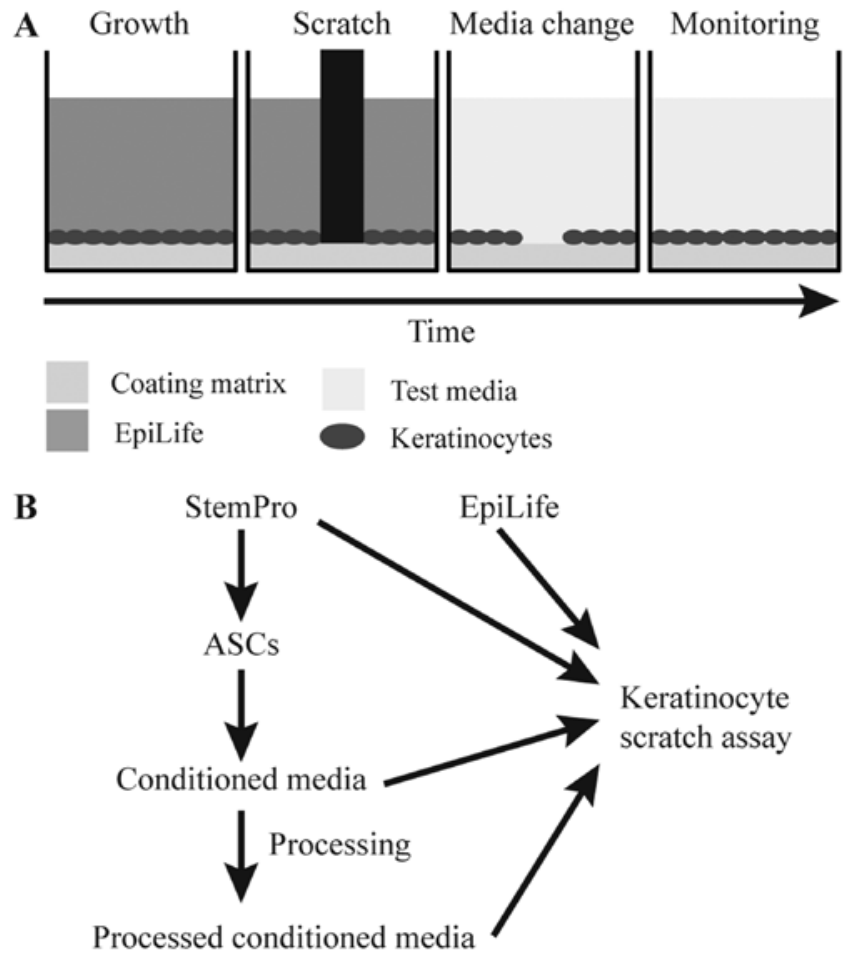

Figure 1. Schematic overview of the scratch assay and the production, preparation and functional testing of conditioned media. (A) Human primary keratinocytes were cultured until confluent in EpiLife, whereupon a pin tool was used to apply a uniform scratch. The medium was replaced with conditioned medium and the process of wound closure monitored to assess the degree of wound healing. (B) To produce conditioned media, StemPro was added to near-confluent adipose-derived stem cell (ASC) cultures. After $24 \mathrm{~h}$, the conditioned StemPro was collected for downstream processing and application in the scratch assay.

StemPro, or EpiLife were added to the cells (Fig. 1B). Wound healing was monitored by time-lapse photography taking images every hour using a PeCon Incubator system including a CTI-controller 3700 digital and a Tempcontrol 37-2 digital, connected to an AxioVision software package with a Zeiss AxioObserver.Z1 microscope equipped with an AxioCam MRm camera and a motorized stage (Carl Zeiss) or using the IncuCyte $\mathrm{ZOOM}^{\circledR}$ system (Essen BioScience). The relative wound size at each time point was analyzed using the TScratch software (20) or the IncuCyte ${ }^{\mathrm{TM}}$ Scratch Wound Cell Migration Software Module (Essen BioScience). The media was tested on three primary keratinocyte cultures in two separate experiments $(n=6)$, each in technical triplicates.

Statistical analysis. Statistical analysis was performed using SigmaPlot 12.0 (Systat Software, Erkrath, Germany). The normal distribution of each group was assessed by means of the Shapiro-Wilk test. Additionally, variance was tested using an Equal Variance test. Data are presented as the mean \pm standard error of the mean (SEM). A p-value $<0.05$ was considered to indicate a statistically significant difference. For the comparison of two groups, a paired t-test was used. For the comparison of more than two groups, a one-way repeated measures analysis of variance (ANOVA) with Bonferroni's post hoc test was used.

\section{Results}

Comparison of the effect of varying calcium concentrations on the morphology and growth of keratinocytes and ASCs. EpiLife and StemPro were evaluated for their compatibility for the culture of both cell types (Fig. 2A). It was evident that the keratinocytes cultured in StemPro lost the typical cobblestone-like appearance of basal keratinocytes and displayed a more mature phenotype with morphology and rearranged cytoskeleton with cortical actin bundles resembling those of differentiated keratinocytes (21). On the other hand, the ASCs retained their morphology when cultured in EpiLife; however, since the number of cells after $24 \mathrm{~h}$ in EpiLife was significantly lower than that in StemPro, it suggested that the growth conditions were sub-optimal. To confirm that these observations were a result of the cellular response to differences in calcium concentrations, we performed a calcium dose escalation experiment with EpiLife medium. For the keratinocytes, a gradual change in cell morphology and cytoskeleton pattern was observed with the increasing calcium concentrations already after $24 \mathrm{~h}$ (Fig. 2B). The cells appeared more clustered and the actin fibers were more diffuse. These morphological changes were slightly noticeable when $120 \mu \mathrm{M}$ calcium were supplemented to the media, and were obviously prominent at concentrations of $480 \mu \mathrm{M}$ and above. No apparent morphological or cytoskeletal effects of changing the calcium concentration were observed for the ASCs. Despite the dramatic effects on keratinocyte morphology, changes in the calcium concentration did not affect cell numbers during short-term exposure (Fig. 2C). This is in sharp contrast to the ASCs, where the use of EpiLife decreased the cell number. This inhibition was attenuated by supplementing EpiLife with calcium in the range of $860 \mu \mathrm{M}$ to $1.4 \mathrm{mM}$ (Fig. 2D).

Development of keratinocyte-compatible media based on StemPro. To circumvent the media incompatibility issue, we cultured ASCs in StemPro for the production of conditioned media. Consequently, for the subsequent use of conditioned media on keratinocytes, we evaluated different methods to reduce the calcium concentration while maintaining the ASC secreted proteins.

A simple solution of culturing the keratinocytes at 3:1, 1:1 and 1:3 ratios of EpiLife to StemPro was tested (Fig. 3A). The evaluation of the morphology revealed that even in the highest dilution of StemPro (with approximately $300 \mu \mathrm{M}$ of calcium), the cultures revealed the presence of clustered keratinocytes, which had lost their cobblestone appearance.

Another method tested was concentrating the protein fraction of StemPro using spin filter columns and afterwards resuspending it in EpiLife in volumes, such that the concentration of proteins derived from StemPro were 50, 75 and $100 \%$ of the original (Fig. 3B). The cells did not display the morphology associated with a high calcium content; however, the cells appeared more round and smaller. In particular, the cells grown in the highest concentration of protein detached before a scratch assay could be completed (data not shown).

The effect of dialyzing StemPro in EpiLife prior to the culture of keratinocytes was examined. We observed that the morphology of the keratinocytes cultured in dialyzed StemPro closely resembled that of cells grown in EpiLife (Fig. 3C, 
A

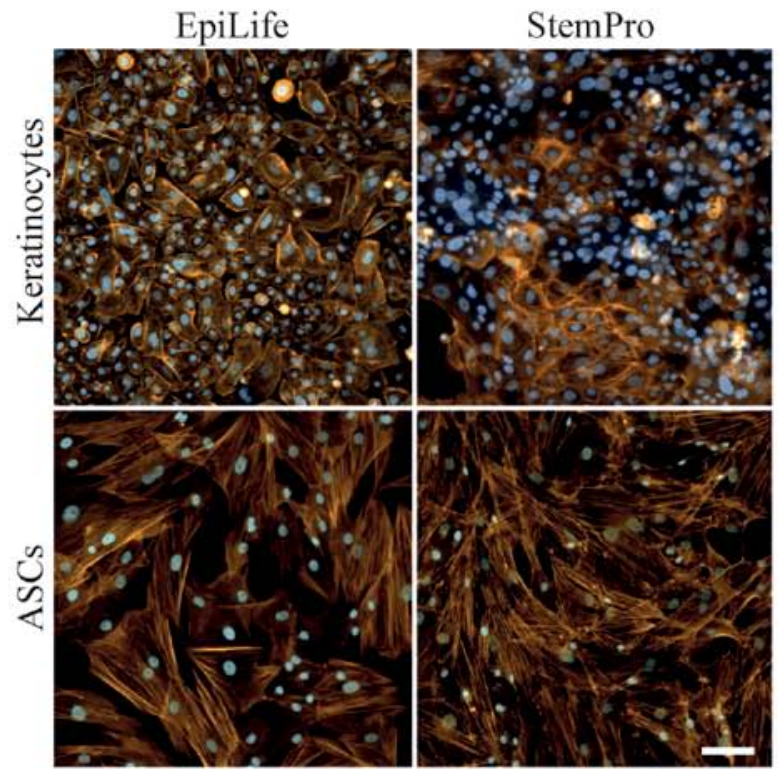

B

0.12

EpiLife $+\mathrm{Ca}^{2+}(\mathrm{mM})$

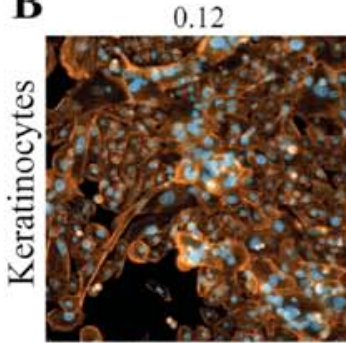

0.48
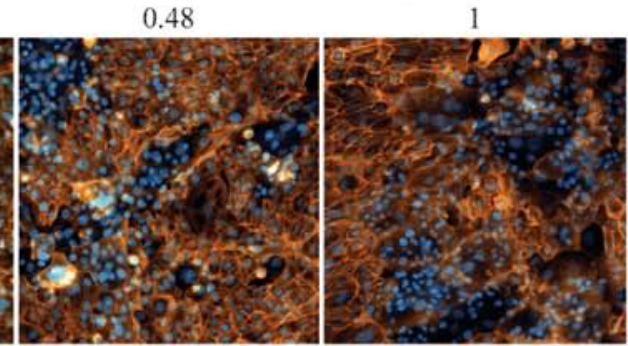

1.6
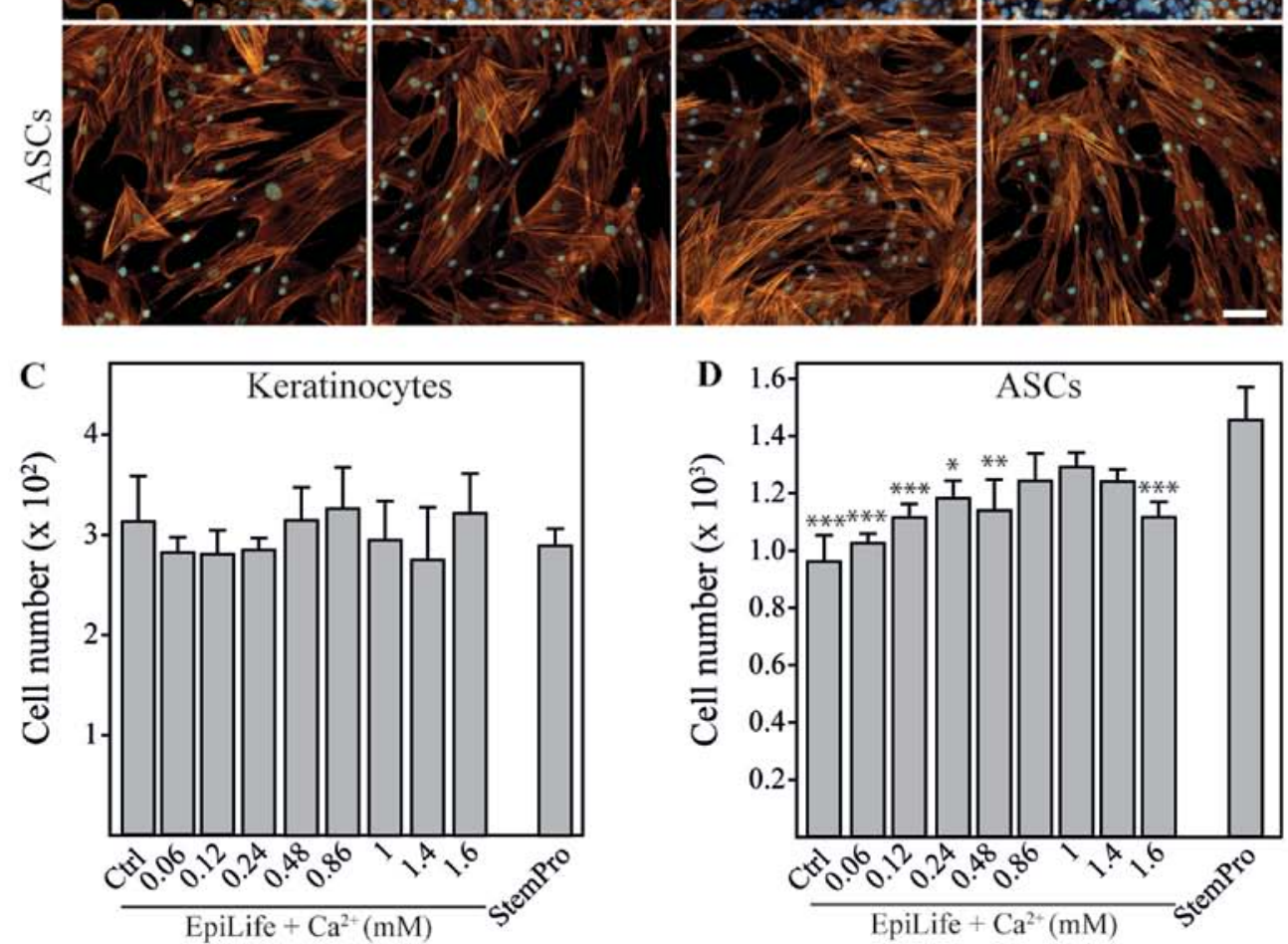

Figure 2. The effect of calcium on morphology and cell number of keratinocytes and adipose-derived stem cells (ASCs). Keratinocytes and ASCs were cultured until $80 \%$ confluent, followed by $24 \mathrm{~h}$ of incubation in EpiLife, EpiLife supplemented with calcium, or StemPro. (A) Representative images of keratinocytes and ASCs showing morphology and cell density after incubation in EpiLife or StemPro. To assess cell morphology and cell number, cytoskeleton were visualized by phalloidin-Bodipy 558/568 staining (orange) and nuclei were counterstained with Hoechst 33342 (blue). The scale bars denote $100 \mu \mathrm{m}$. (B) Representative images of keratinocytes and ASCs showing morphology and cell density after incubation in EpiLife supplemented with increasing concentrations of calcium. Assessment of morphology was performed as described for (A). (C) Number of keratinocytes after incubation in EpiLife (Ctrl), EpiLife supplemented with increasing concentrations of calcium, or StemPro. Values are represented as the means and SEM; no statistically significant differences were found ( $\mathrm{n}=6$ ). (D) Number of ASCs after incubation in EpiLife, EpiLife supplemented with increasing concentrations of calcium, or StemPro. Values are represented as mean and SEM $(\mathrm{n}=6)$. The data from the EpiLife-based media were compared to those from StemPro using a one-way ANOVA followed by a multiple comparisons vs. control, ${ }^{*} \mathrm{p}<0.05,{ }^{* *} \mathrm{p}<0.01$ and ${ }^{* * *} \mathrm{p}<0.001$. 
A

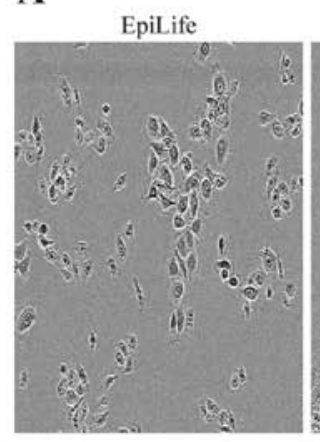

B

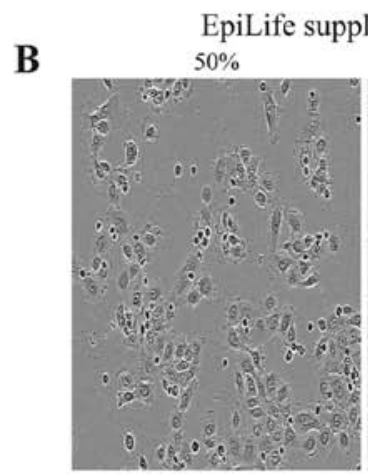

C
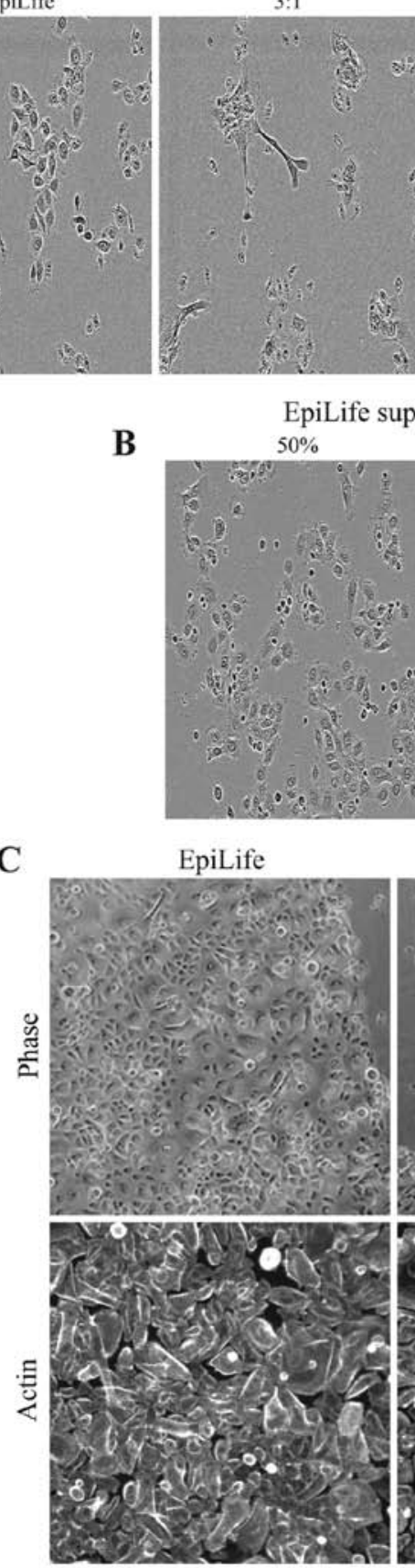

EpiLife/StemPro
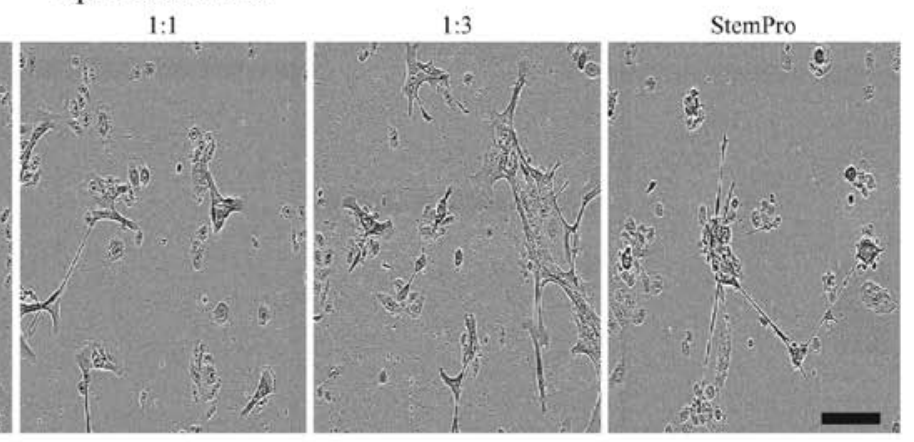

StemPro
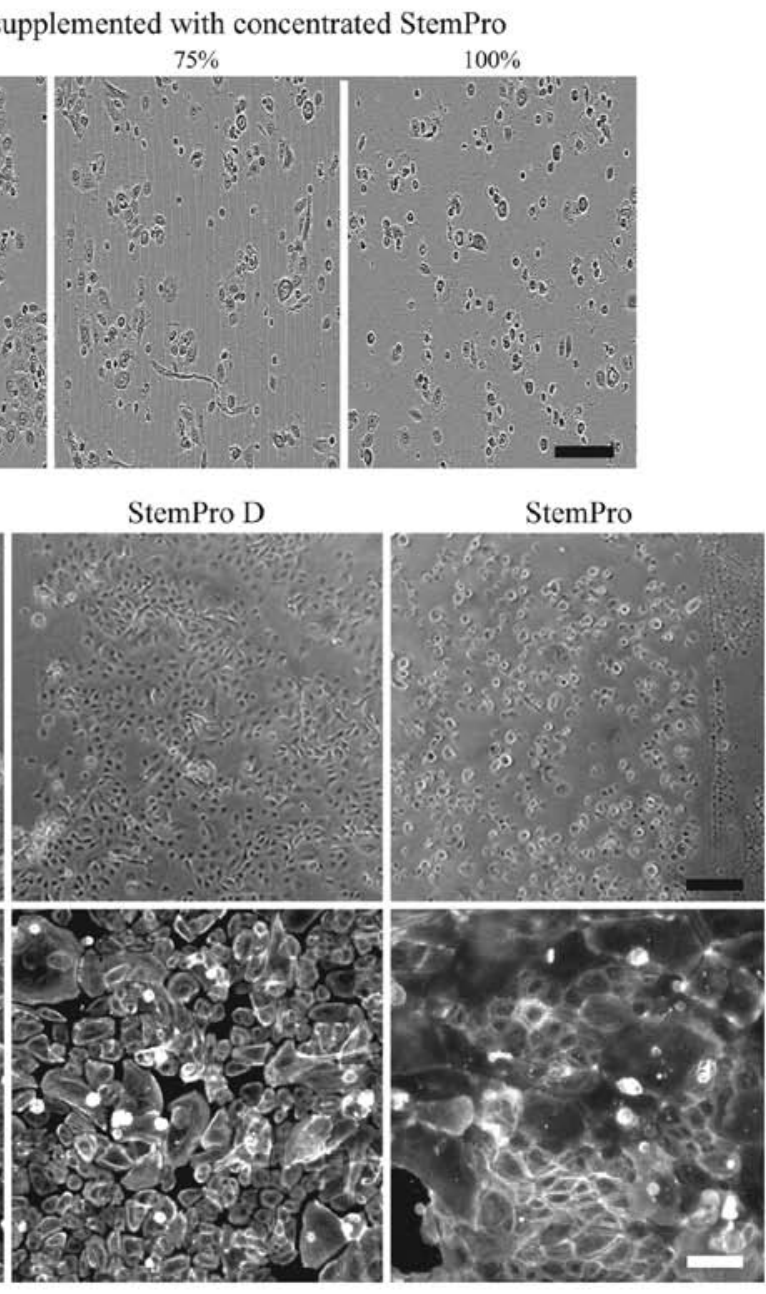

Figure 3. Effect on keratinocyte morphology of $24 \mathrm{~h}$ culture in combinations of EpiLife and StemPro. (A) Keratinocytes were cultured in either EpiLife, StemPro, or combinations of EpiLife and StemPro in the ratios of 3:1, 1:1 and 1:3, after which the morphology was assessed by phase contrast microscopy. The scale bars denote $150 \mu \mathrm{m}$. (B) The protein fraction from conditioned StemPro was concentrated on spin columns, reconstituted in EpiLife at 50,75 and $100 \%$ of the original concentration, and added to keratinocytes. The morphology was assessed by phase contrast microscopy as above. (C) Keratinocytes were cultured in either EpiLife, StemPro dialyzed against EpiLife (StemPro D), or StemPro. The morphology was assessed by phase contrast microscopy. The scale bars denote $200 \mu \mathrm{m}$. To assess the cytoskeleton, actin fibers were visualized by fluorescence microscopy using a phalloidin-Bodipy $558 / 568$ staining. The scale bars denote $100 \mu \mathrm{m}$.

upper panel). Additionally, the visualization of the cytoskeleton revealed that StemPro induced a more peripheral arrangement of the actin fibers, and that this to a high degree was avoided by using dialyzed StemPro that maintained the diffuse pattern (Fig. 3C, lower panel).

Evaluation of keratinocyte-compatible media based on StemPro in a scratch assay. To functionally assess the kera- tinocyte-compatible conditioned and dialyzed StemPro, this media was compared to high-calcium media (StemPro and conditioned StemPro) and low-calcium non-conditioned media (dialyzed StemPro and EpiLife) in a scratch assay (Fig. 4). The conditioned and dialyzed StemPro increased the closure rate of the scratch when compared to the low-calcium nonconditioned media. The two low-calcium non-conditioned media (dialyzed StemPro and EpiLife) both supported healing 

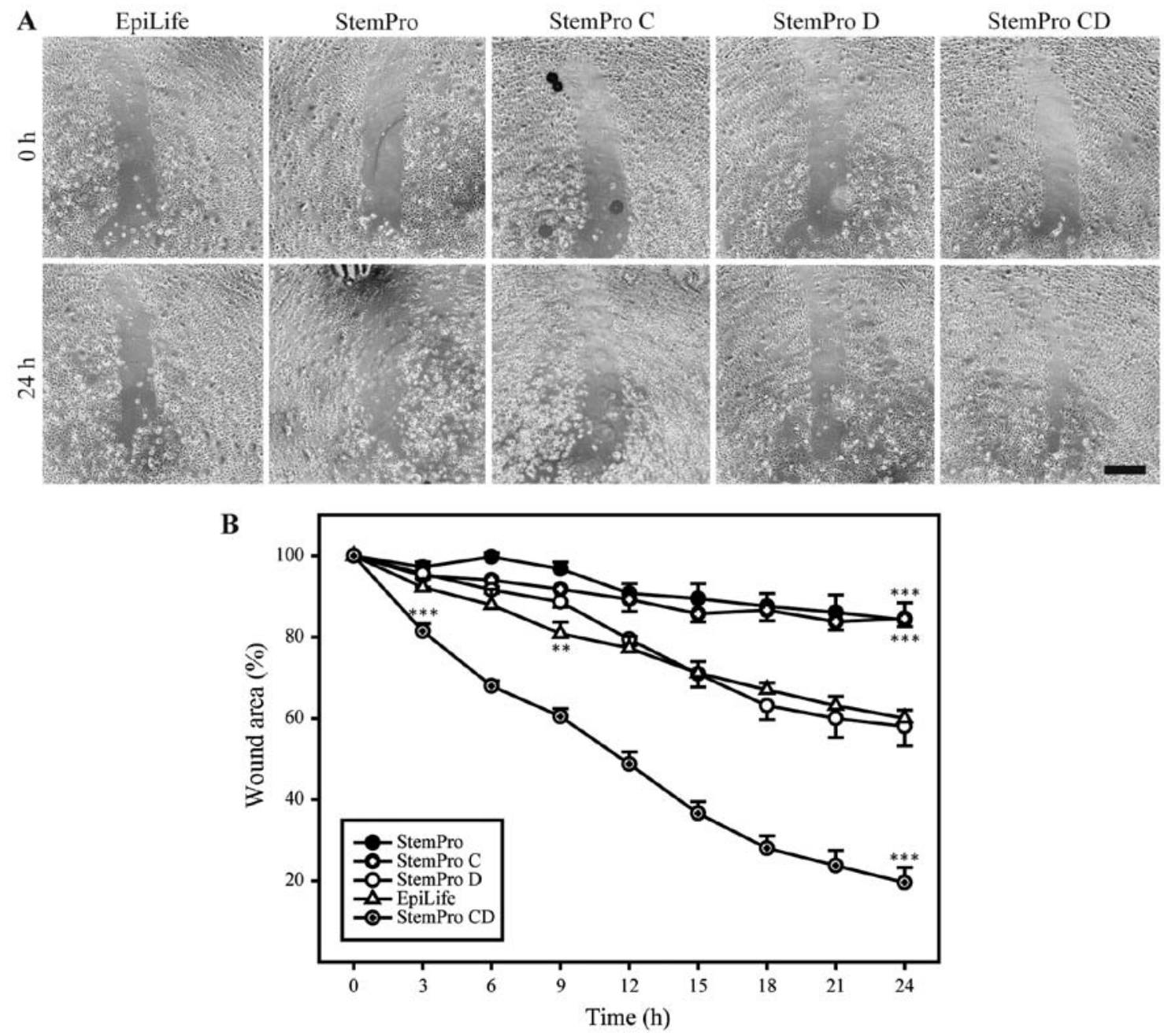

Figure 4. Effect of different media on the wound healing of keratinocytes. (A) Representative micrographs from each condition, showing the original wound and the wound after $24 \mathrm{~h}$. Scale bar denotes $500 \mu \mathrm{m}$. (B) Quantitative analysis of wound closure as a function of time. The wound area was determined as the wound area at a given time relative to the original wound area. Data are presented as the means \pm SEM. The dialyzed conditioned StemPro (StemPro CD) consistently supported wound healing better than EpiLife, and the difference in performance was statistically significant at $3 \mathrm{~h}$ after wounding $(\mathrm{p}<0.001)$ and onwards. Additionally, the non-dialyzed StemPro media performed worse than EpiLife, and this difference was statistically significant from $9 \mathrm{~h}$ after wounding ( $<<0.01$, $\mathrm{n}=6) .{ }^{* *} \mathrm{p}<0.01$ and $^{* * *} \mathrm{p}<0.001$.

of the scratch in a comparable manner. Furthermore, the results from the non-dialyzed StemPro media confirmed the unsuitability of using high-calcium media when assessing growth and migration of primary keratinocytes.

Effect of hypoxia on the wound healing properties of ASCs. The dialyzed conditioned media derived from either ASCs cultured under either hypoxic or normoxic conditions was compared in the keratinocyte scratch assay. No differences between the media were observed with respect to the effect on cell morphology. However, it appeared that the closure of the scratch was more complete in the keratinocytes exposed to the media from ASCs cultured under hypoxic conditions (Fig. 5A). For both types of media, the repopulation of the wound area occurred through cells migrating in an individual random pattern, rather than as linear fronts of the wound edge approximating one another. A quantification of the keratinocyte wound closure in the conditioned media compared to the closure in EpiLife, revealed that both normoxic and hypoxic conditioned media promoted wound closure to a greater extent than EpiLife (data not shown). Furthermore, it appeared that the media from the ASCs cultured under hypoxic conditions was consistently superior to that from the ASCs cultured under normoxic conditions, and this difference was statistically significant after $24 \mathrm{~h}$ (Fig. 5B).

\section{Discussion}

For the development of a treatment for chronic wounds, it is apparent from animal experiments and small-scale clinical trials that ASCs harbor a significant potential for use in regenerative therapy (22). To harness that potential, good in vitro models are warranted that enable the systematic analysis of the various wound healing properties of ASCs and how these properties may be enhanced.

In the present study, we described the modification of a scratch assay, such that an evaluation of the ASC secretome on wound healing properties of human primary keratinocytes could be carried out. To create a scratch assay that is compatible with both ASCs and primary keratinocytes, we tried a number of approaches. First, we explored whether both ASCs and keratinocytes could be cultured in either EpiLife or StemPro, 
A
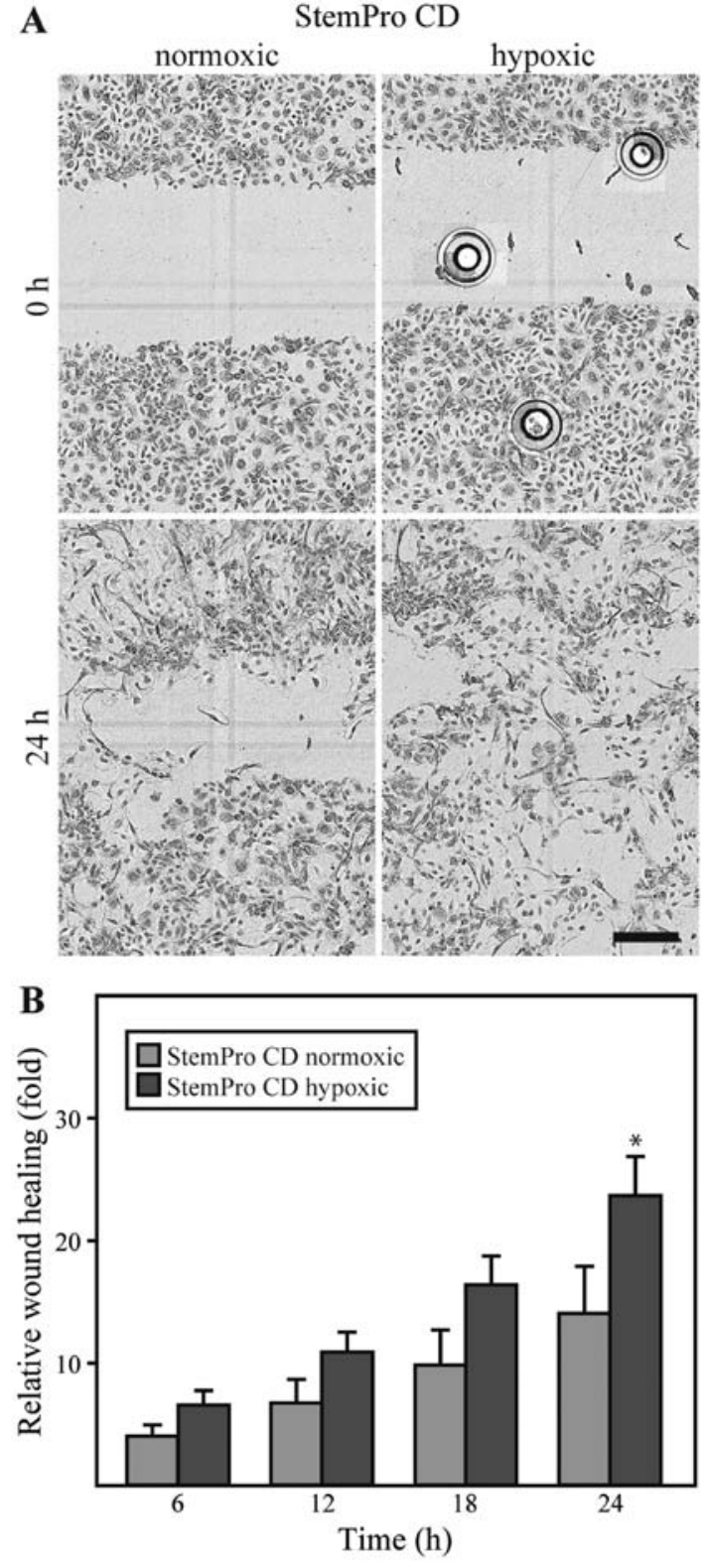

Figure 5. Effect of hypoxic preconditioning on the wound healing properties of adipose-derived stem cells (ASCs). Conditioned and dialyzed StemPro (StemPro CD) from ASCs cultured either under normoxic or hypoxic conditions, was used in the scratch assay on keratinocytes. (A) Representative micrographs from each condition, showing the original wound and the wound after $24 \mathrm{~h}$ Scale bar denotes $300 \mu \mathrm{m}$. The circular shapes in the upper right image are air bubbles from the scratching procedure. (B) Quantitative analysis of relative wound closure at different time points. First, the wound closure was determined as the wound area at a given time relative to the original wound area, after which the relative wound closure was calculated as wound closure in conditioned medium divided by the wound closure in EpiLife. Data are presented as the means $\pm \operatorname{SEM}(\mathrm{n}=6)$. The data from normoxic and hypoxic media was compared showing a statistical significant difference after $24 \mathrm{~h},{ }^{*} \mathrm{p}<0.01$.

such that the conditioned medium from the ASCs could be use directly on the keratinocytes. Since the ASCs did not proliferate in EpiLife, confirming previous observations of ASCs in keratinocyte media (23) and the keratinocytes differentiated in StemPro, this option was not viable. As the literature describes keratinocyte media to be low and mesenchymal stem cell media to be high in calcium (14), we speculated that the failure of either cell type to thrive in the medium developed for the other could be caused by different requirements for calcium.
A titration of calcium for both cell types confirmed this. The primary keratinocytes differentiated when exposed to even moderately higher calcium concentrations than those found in EpiLife, in agreement with what is already known (24), and the ASCs cell number decreased when the calcium concentration was lower than that found in standard ASC media, possibly due to the influence of calcium on the cell adhesion to the substrate.

Based on the incompatibility of the media with both cell types, several approaches were explored to decrease the calcium contribution from the conditioned StemPro. Simple dilution of StemPro with EpiLife (up to 1:3) did not decrease the calcium concentration sufficiently to avoid differentiation of the keratinocytes. Further dilution was not attempted due to the risk of attenuating the putative effect of the ASCs. Re-suspending a concentrated protein fraction of the conditioned StemPro in EpiLife was not a solution either, as the keratinocytes did not attach well in this media, possibly due to increases in osmolarity. When StemPro was dialyzed, a normal keratinocyte morphology was maintained. Furthermore, we observed that dialyzed StemPro and EpiLife supported keratinocyte migration in an equivalent manner. Thus, it is possible to assess the wound healing effects of ASCs in a scratch assay with primary keratinocytes, provided that the conditioned media is dialyzed.

When applying conditioned media from ASCs cultured under normoxic or hypoxic conditions, it was obvious that the ASCs secreted factors that promoted primary keratinocyte wound healing in vitro, and that this effect was enhanced by culturing the ASCs under hypoxic conditions. Our results are thus consistent with the findings of other studies, in that ASCs and other mesenchymal stem cells promote keratinocyte-based wound healing significantly $(3,8)$. Furthermore, our findings indicated that this effect is also present when studying the more relevant primary human keratinocytes rather than immortalized cell lines. Of note, the random migratory pattern of the cells during wound closure could be an indication of the keratinocytes undergoing a epithelial-to-mesenchymal transition (25).

In general, mesenchymal cells are known to provide a microenvironment that maintains the progenitor status of basal keratinocytes and augments epidermal proliferation, and mesenchymal cells are therefore commonly used as feeder cells in keratinocyte cultures (26). In connection to this, ASCs, which are of mesenchymal origin, have been shown to secrete a plethora of growth factors, such as keratinocyte growth factor (KGF), EGF, bFGF, hepatocyte growth factor (HGF), and insulin-like growth factor-1 (IGF-1) (27), which are key mediators of migration of human primary keratinocytes (28). The secretion of these factors could provide part of the explanation for the effect of the conditioned medium on keratinocyte wound closure.

When using conditioned media from ASCs cultured under hypoxic conditions, an even more pronounced effect on the wound closure of the keratinocytes was observed. The exposure of ASCs to hypoxia has been shown to increase the secretion of a wide range of growth factors, signaling molecules and cytokines (29). bFGF and IGF-1 are among these factors $(30,31)$, and it is possible that these proteins play a role in the enhanced stimulatory effect of the hypoxic-conditioned ASCs. The majority of studies on the ASC secretome under hypoxic conditions have focused on a narrow range of proteins mostly involved in angiogenesis $(2,4,16)$, and not on global discovery-based approaches. To begin to unravel the molecular 
mechanisms underlying the wound healing effects of ASCs, we recently performed a proteomic profiling of ASCs exposed to hypoxia and normoxia, and found that several of the factors differentially regulated by hypoxia were involved in extracellular matrix (ECM) synthesis (32). It will be interesting to determine whether the ECM-related proteins secreted by ASCs are responsible for the observed effects on the keratinocytes.

The establishment of good wound models will support the translation of ASCs into clinical use, as they can play an important role both for the development and validation of novel protocols prior to embarking on large-scale clinical studies (33). Additionally, the models may be used post-translationally to predict treatment outcome, so forth a correlation between the modeled parameter and the clinical outcome can be verified. Good wound models may thus facilitate the translation of regenerative ASC-based wound therapies into clinical practice.

\section{Acknowledgements}

The authors acknowledge the technical assistance provided by O. Jensen and L. Sangenario. This study was supported in part by funds from Lily Benthine Lunds fond (S.R.), Grosserer L.F. Foghts Fond (V.Z.) and the Obelske family foundation (T.F.). The funding sources had no influence on either the study design, collection, analysis, interpretation of the data, the writing of the study, or on the decision to submit the study for publication. R.N., D.K., S.B. and M.V. are regular employees of ThermoFisher Scientific and have not received any financial gains. They hold some stocks of ThermoFisher Scientific as employees of ThermoFisher Scientific.

\section{References}

1. Hassan WU, Greiser U and Wang W: Role of adipose-derived stem cells in wound healing. Wound Repair Regen 22: 313-325, 2014.

2. Rasmussen JG, Frøbert O, Pilgaard L, Kastrup J, Simonsen U, Zachar V and Fink T: Prolonged hypoxic culture and trypsinization increase the pro-angiogenic potential of human adipose tissue-derived stem cells. Cytotherapy 13: 318-328, 2011.

3. Lee SH, Jin SY, Song JS, Seo KK and Cho KH: Paracrine effects of adipose-derived stem cells on keratinocytes and dermal fibroblasts. Ann Dermatol 24: 136-143, 2012.

4. Rehman J, Traktuev D, Li J, Merfeld-Clauss S, Temm-Grove CJ Bovenkerk JE, Pell CL, Johnstone BH, Considine RV and March KL: Secretion of angiogenic and antiapoptotic factors by human adipose stromal cells. Circulation 109: 1292-1298, 2004.

5. Hsiao ST, Lokmic Z, Peshavariya H, Abberton KM, Dusting GJ, Lim SY and Dilley RJ: Hypoxic conditioning enhances the angiogenic paracrine activity of human adipose-derived stem cells. Stem Cells Dev 22: 1614-1623, 2013.

6. Pastar I, Stojadinovic O, Yin NC, Ramirez H, Nusbaum AG, Sawaya A, Patel SB, Khalid L, Isseroff RR and Tomic-Canic M: Epithelialization in Wound Healing: A comprehensive review. Adv Wound Care (New Rochelle) 3: 445-464, 2014.

7. Miranda JP, Filipe E, Fernandes AS, Almeida JM, Martins JP, De la Fuente A, Abal M, Barcia RN, Cruz P, Cruz H, et al: The human umbilical cord tissue-derived MSC population UCX(®) promotes early motogenic effects on keratinocytes and fibroblasts and G-CSF-mediated mobilization of BM-MSCs when transplanted in vivo. Cell Transplant 24: 865-877, 2015.

8. Walter MN, Wright KT, Fuller HR, MacNeil S and Johnson WE: Mesenchymal stem cell-conditioned medium accelerates skin wound healing: An in vitro study of fibroblast and keratinocyte scratch assays. Exp Cell Res 316: 1271-1281, 2010.

9. Hanahan D and Weinberg RA: Hallmarks of cancer: The next generation. Cell 144: 646-674, 2011.

10. Zhang J, Antonyak MA, Singh G and Cerione RA: A mechanism for the upregulation of EGF receptor levels in glioblastomas. Cell Reports 3: 2008-2020, 2013.
11. Ahmad I, Iwata T and Leung HY: Mechanisms of FGFR-mediated carcinogenesis. Biochim Biophys Acta 1823: 850-860, 2012.

12. Turunen A and Syrjänen S: Extracellular calcium regulates keratinocyte proliferation and HPV 16 E6 RNA expression in vitro. APMIS 122: 781-789, 2014

13. Micallef L, Belaubre F, Pinon A, Jayat-Vignoles C, Delage C, Charveron $\mathrm{M}$ and Simon A: Effects of extracellular calcium on the growth-differentiation switch in immortalized keratinocyte HaCaT cells compared with normal human keratinocytes. Exp Dermatol 18: 143-151, 2009.

14. Conrad DR: Calcium in Cell Culture. Technical note. Sigma-Aldrich. http://www.sigmaaldrich.com/life-science/cellculture/learning-center/media-expert/calcium.html.

15. Zachar V, Rasmussen JG and Fink T: Isolation and growth of adipose tissue-derived stem cells. Methods Mol Biol 698: 37-49, 2011.

16. Rasmussen JG, Riis SE, Frøbert O, Yang S, Kastrup J, Zachar V, Simonsen U and Fink T: Activation of protease-activated receptor 2 induces VEGF independently of HIF-1. PLoS One 7: e46087, 2012.

17. Yang S, Pilgaard L, Chase LG, Boucher S, Vemuri MC, Fink T and Zachar V: Defined xenogeneic-free and hypoxic environment provides superior conditions for long-term expansion of human adipose-derived stem cells. Tissue Eng Part C Methods 18: 593-602, 2012.

18. Fink T, Rasmussen JG, Lund P, Pilgaard L, Soballe K and Zachar V: Isolation and expansion of adipose-derived stem cells for tissue engineering. Front Biosci (Elite Ed) 3: 256-263, 2011.

19. Prasad M, Zachar V, Fink T and Pennisi CP: Moderate hypoxia influences potassium outward currents in adipose-derived stem cells. PLoS One 9: e104912, 2014.

20. Gebäck T, Schulz MMP, Koumoutsakos P and Detmar M: TScratch: A novel and simple software tool for automated analysis of monolayer wound healing assays. Biotechniques 46: 265-274, 2009.

21. Vespa A, Darmon AJ, Turner CE, D'Souza SJA and Dagnino L: $\mathrm{Ca}^{2+}$-dependent localization of integrin-linked kinase to cell junctions in differentiating keratinocytes. J Biol Chem 278: 11528-11535, 2003.

22. Cerqueira MT, Pirraco RP and Marques AP: Stem cells in skin wound healing: Are we there yet? Adv Wound Care (New Rochelle) 5: 164-175, 2016.

23. Seo BF, Kim KJ, Kim MK and Rhie JW: The effects of human keratinocyte coculture on human adipose-derived stem cells. Int Wound J 13: 630-635, 2014.

24. Bikle DD, Xie Z and Tu CL: Calcium regulation of keratinocyte differentiation. Expert Rev Endocrinol Metab 7: 461-472, 2012.

25. Moreno-Bueno G, Peinado H, Molina P, Olmeda D, Cubillo E, Santos V, Palacios J, Portillo F and Cano A: The morphological and molecular features of the epithelial-to-mesenchymal transition. Nat Protoc 4: 1591-1613, 2009.

26. Werner S, Krieg T and Smola H: Keratinocyte-fibroblast interactions in wound healing. J Invest Dermatol 127: 998-1008, 2007.

27. Kapur SK and Katz AJ: Review of the adipose derived stem cell secretome. Biochimie 95: 2222-2228, 2013.

28. Peplow PV and Chatterjee MP: A review of the influence of growth factors and cytokines in in vitro human keratinocyte migration. Cytokine 62: 1-21, 2013.

29. Zachar V, Duroux M, Emmersen J, Rasmussen JG, Pennisi CP, Yang S and Fink T: Hypoxia and adipose-derived stem cell-based tissue regeneration and engineering. Expert Opin Biol Ther 11: 775-786, 2011.

30. Liu L, Gao J, Yuan Y, Chang Q, Liao Y and Lu F: Hypoxia preconditioned human adipose derived mesenchymal stem cells enhance angiogenic potential via secretion of increased VEGF and bFGF. Cell Biol Int 37: 551-560, 2013.

31. An HY, Shin HS, Choi JS, Kim HJ, Lim JY and Kim YM: Adipose mesenchymal stem cell secretome modulated in hypoxia for remodeling of radiation-induced salivary gland damage. PLoS One 10: e0141862, 2015.

32. Riis S, Stensballe A, Emmersen J, Pennisi CP, Birkelund S, Zachar V and Fink T: Mass spectrometry analysis of adiposederived stem cells reveals a significant effect of hypoxia on pathways regulating extracellular matrix. Stem Cell Res Ther 7: $52,2016$.

33. Riis S, Zachar V, Boucher S, Vemuri MC, Pennisi CP and Fink T: Critical steps in the isolation and expansion of adipose-derived stem cells for translational therapy. Expert Rev Mol Med 17: e11, 2015. 